Article

\title{
Rendering the Navier-Stokes Equations for a Compressible Fluid into the Schrödinger Equation for Quantum Mechanics
}

\author{
Peter Vadasz
}

Department of Mechanical Engineering, Northern Arizona University, P.O. Box 15600, Flagstaff, AZ 86001, USA; peter.vadasz@nau.edu; Tel.: +1-928-523-5843

Academic Editor: Rama S.R. Gorla

Received: 2 May 2016; Accepted: 6 June 2016; Published: 13 June 2016

\begin{abstract}
The mass and momentum transfer phenomena in a compressible fluid represented by the Navier-Stokes equations are shown to convert into the Schrödinger equation for quantum mechanics. The complete Navier-Stokes equations render into an extended generalized version of Schrödinger equation. These results complement the Madelung's (Zeitschrift für Physik 40 (3-4), pp. 322-326, 1926-1927) derivations that show how Schrödinger's equation in quantum mechanics can be converted into the Euler equations for irrotational compressible flow. The theoretical results presented here join the classical Madelung paper to suggest the possibility that quantum effects at sub-atomic levels deal with a compressible fluid susceptible to wave propagation, rather than a particle. The link between such a fluid and the "quantum particle" is under current investigation.
\end{abstract}

Keywords: Navier-Stokes equations; mass and momentum transfer; Schrödinger equation; quantum mechanics; Madelung model

PACS: J0101

\section{Introduction}

(A large number of references presented in this paper are originally published in the German language. For readers not familiar with, or not fluent in German, such references are always followed by associated references of the same papers translated into English.)

Quantum theory governs all phenomena at the sub-atomic scale. It evolved into a probabilistic theory, and its weird effects were over the years a matter of fascination, and its interpretations a matter of much controversy. While the quantitative results were never disputed, their physical interpretation caused an overwhelming debate between the leading 20th century scientists. Following Bohm [1], Griffiths [2], and Bowman [3] the kernel of quantum mechanics is the Schrödinger wave equation [4-8]. Born [9] introduced a statistical interpretation to the wave-function appearing in the Schrödinger equation to Erwin Schrödinger's explicit disapproval [4-8] and annoyance, who preferred the wave field interpretation. Eventually the statistical approach was entrenched in quantum mechanics not only as a technical means of providing answers and solutions to sub-atomic phenomena but as a "complete" interpretation of the physical "reality" following Bohr's and Heisenberg's "Copenhagen Interpretation" that became main stream Physics. The latter was challenged not only by Schrödinger but also by a large group of physicists led by Albert Einstein who claimed that the quantum mechanical description of the physical reality cannot be considered complete, as shown in their famous EPR paper Einstein, Podolsky and Rosen [10]. They concluded their derivations by stating that "While we have thus shown that the wave function does not provide a complete description of the physical reality, we left open the question of whether or not such a description exists. We believe, however, 
that such a theory is possible." Einstein did not object to the probabilistic description of sub-atomic phenomena in quantum mechanics. However, he believed that this probabilistic representation was a technique used to overcome the practical difficulties of dealing with a more complicated underlying physical reality, much in the same way he suggested earlier to deal with Brownian motion [11,12]. Niels Bohr led another group of physicists including Werner Heisenberg, Max Born, Wolfgang Pauli and others that believed in the probabilistic interpretation of sub-atomic phenomena as being complete. Bohr's answer [13] to the EPR paper [10] was linked to the fact that the finite interaction between an object and the measuring device "entails — because of the impossibility of controlling the reaction of the object on the measuring instruments ... - the necessity of a final renunciation of the classical ideal of causality and a radical revision of our attitude towards the problem of physical reality" [13]. The latter is linked to the concept of "complementarity" [13].

Madelung $[14,15]$ showed that the Schrödinger equation could be rendered into an equation very similar to the Euler equations and applicable to potential flow. Sonego [16] attempted to provide an interpretation of the "hydrodynamical formalism of quantum mechanics" that is consistent with the probabilistic Copenhagen interpretation and therefore negating the possibility of physical fluid dynamical meanings of the Madelung variables. Takabayasi $[17,18]$ presented the Madelung equations as an ensemble of trajectories. Wilhelm [19] investigated the hydrodynamic formulation of quantum mechanics with emphasis "on the physical peculiarities appearing in the hydrodynamic picture." Sorokin [20] further investigated the Madelung transformations for vortex flows of a perfect fluid. Broadbridge [21] extended the scalar wave function in the Schrödinger equation to a vector potential by using the Helmholtz decomposition of the Madelung fluid that included a solenoidal component. All these studies focused on starting from the Schrödinger equation and leading to the Madelung form of Euler equations for potential flow that was analyzed further. The present paper starts from the Navier-Stokes equations and shows how the latter convert into a generalized form of Schrödinger equation.

\section{Problem Formulation and the Madelung Transformation}

The Schrödinger equation is a linear partial differential equation that has the form

$$
i \hbar \frac{\partial \psi}{\partial t}=-\frac{\hbar^{2}}{2 m} \nabla^{2} \psi+V(\mathbf{x}) \psi
$$

where $\psi$ is the complex wave function, and $\mathbf{x}=x \hat{\mathbf{e}}_{\mathbf{x}}+y \hat{\mathbf{e}}_{\mathbf{y}}+z \hat{\mathbf{e}}_{\mathbf{z}}$ is the position vector in Cartesian coordinates, $t$ is time, $V(\mathbf{x})$ is a potential function due to conservative forces, $m$ is the quantum particle mass considered constant, $\hbar=h / 2 \pi$ is the reduced Plank's constant, and $i=\sqrt{-1}$. The solution to the Schrödinger equation depends on the imposed potential $V(\mathbf{x})$ but generally has a wave solution that in the one dimensional finite space takes the form

$$
\psi(x, t)=\sum_{n=1}^{\infty} C_{n} \phi_{n}(x) \exp \left(-i \frac{E_{n}}{\hbar} t\right)
$$

where

$$
C_{n}=\int \phi_{n}^{*}(x) \psi_{o}(x) d x
$$

where $\phi_{n}^{*}$ is the complex conjugate of $\phi_{n}, \psi_{o}(x)=[\psi(x, t)]_{t=0}=\psi(x, 0)$ is the initial condition for the wave function, and $E_{n}$ is the total energy associated with mode (quantum number) $n$. The solution (2) to the Schrödinger equation for the complex wave-function $\psi$ leads to the superposition of an infinite number of modes, while Born [9] interpretation implies that

$$
\left.\int_{a}^{b} \psi \psi^{*} \mathrm{~d} x=\text { [probability to find a particle in the interval between } a \text { and } b\right]
$$


where $\psi^{*}$ is the complex conjugate of $\psi$. Whatever the interpretation, the experimental fact still shows that the wave-function collapses upon observation, i.e., as soon as experimental observation (e.g., measurement) is undertaken the wave-function collapses from the superposition of an infinite number of modes, as presented in Equation (2), to one single mode. This is also referred to as "the reduction of the wave packet, i.e., the wave packet given by the infinite series" e.g., (2), "is reduced to a single term" [10].

Madelung $[14,15]$ showed that the Schrödinger equation could be rendered into an equation very similar to the Euler equations and applicable to potential flow. Starting from Schrödinger Equation (1) one may represent the (complex) wave function in the form

$$
\psi(\mathbf{x}, t)=R(\mathbf{x}, t) \exp \left[\frac{i}{\hbar} S(\mathbf{x}, t)\right]
$$

and upon substituting (5) into (1) and using the notation

$$
\rho=R^{2}=\psi \psi^{*} ; S=-i \frac{\hbar}{2} \ln \left(\frac{\psi}{\psi^{*}}\right) ; v=\nabla S=-i \frac{\hbar}{2} \frac{\psi^{*}}{\psi} \nabla\left(\frac{\psi}{\psi^{*}}\right)
$$

one obtains the following set of equations

$$
\begin{gathered}
\frac{\partial \rho}{\partial t}+\nabla \cdot(\rho v)=0 \\
\frac{\partial v}{\partial t}+(v \cdot \nabla) v=\frac{\hbar^{2}}{2 m^{2}} \nabla\left[\frac{\nabla^{2}\left(\rho^{1 / 2}\right)}{\rho^{1 / 2}}\right]+\frac{1}{m} \nabla V .
\end{gathered}
$$

This is the Madelung transformation that renders the Schrödinger equation complex scalar variable $\psi(\mathbf{x}, t)$ into two real variables, one scalar and the other a vector, i.e., $\rho(\mathbf{x}, t)$ and $\mathbf{v}(\mathbf{x}, t)$, respectively. Equations (7) and (8) are very similar with the Euler equations for compressible potential flow

$$
\begin{gathered}
\frac{\partial \rho}{\partial t}+\nabla \cdot(\rho v)=0 \\
\frac{\partial v}{\partial t}+(v \cdot \nabla) v=-\frac{1}{\rho} \nabla p+\nabla\left[g\left(\hat{\mathbf{e}}_{\mathbf{g}} \cdot \mathbf{x}\right)\right]
\end{gathered}
$$

where $v$ is the fluid's velocity vector, $\rho$ is the fluid's mass density, $p$ is the fluid's pressure, and $g$ is the acceleration due to gravity. Note that $\nabla\left(\hat{\mathbf{e}}_{\mathbf{g}} \cdot \mathbf{x}\right)=\hat{\mathbf{e}}_{\mathbf{g}}$, where $\hat{\mathbf{e}}_{\mathbf{g}}$ is a unit vector in the direction of the acceleration due to gravity, and $\mathbf{x}=x \hat{\mathbf{e}}_{\mathbf{x}}+y \hat{\mathbf{e}}_{\mathbf{y}}+z \hat{\mathbf{e}}_{\mathbf{z}}$ is the position vector in Cartesian coordinates. The definition of $v=\nabla S$ from (6) makes this analogy applicable for potential flow only, quite a severe limitation. It is also difficult to relate the potentials in Equation (8) to specific terms in Equation (10), while the pressure gradient can be associated with one component of the potential function $V$ in Equation (8). The other components are potentials from other conservative forces such as Coulomb's electrostatic force or similar, that can be added as body forces on the right-hand-side of Equation (10). Alternatively the pressure gradient can be associated with the gradient term in Equation (8), suggesting a relationship between the density and pressure.

\section{Rendering the Transformation of the Navier-Stokes Equations via Helmholtz Decomposition}

\subsection{Navier-Stokes Equations}

A generalized form of Navier-Stokes equations applicable to compressible fluids subject to pressure forces per unit volume, proportional to $\nabla p$, body forces per unit volume, B, deviatoric 
stresses, $\tau$, and other dissipative forces per unit volume (e.g., Larmor radiation), $\mathbf{D}$, can be presented in the following form

$$
\begin{gathered}
\frac{\partial \rho}{\partial t}+\nabla \cdot(\rho v)=0 \\
\rho\left[\frac{\partial v}{\partial t}+(v \cdot \nabla) v\right]=-\nabla p-\nabla \cdot \underset{\tau}{\tau}+\mathbf{B}+\mathbf{D}
\end{gathered}
$$

where $v$ is the fluid's velocity vector, $\rho$ is the fluid's mass density, and $p$ is the fluid's pressure, $\underset{\sim}{\tau}$ is the deviatoric stress tensor, $\mathbf{B}$ represents all body forces per unit volume including gravity, electromagnetic forces, buoyancy, etc., and $\mathbf{D}$ accounting for all dissipative forces (e.g., Larmor radiation) other than viscous forces, the latter forming part of the deviatoric stress tensor. In other words considering $M$ body forces and $N$ dissipative forces other than the viscous one, leads to

$$
\begin{aligned}
& \mathbf{B}=\sum_{i=1}^{M} \mathbf{B}_{\mathbf{i}}=\rho \sum_{i=1}^{M} \mathbf{b}_{\mathbf{i}}=\rho \mathbf{b} \\
& \mathbf{D}=\sum_{i=1}^{N} \mathbf{D}_{\mathbf{i}}=\rho \sum_{i=1}^{N} \mathbf{d}_{\mathbf{i}}=\rho \mathbf{d}
\end{aligned}
$$

where $\rho$ is fluid's mass density and $\mathbf{b}_{\mathbf{i}}$ is a specific body force (i.e., force per unit mass), e.g., in the case of gravity when the body force is $\mathbf{B}_{\mathbf{g}}=\rho g \hat{\mathbf{e}}_{\mathbf{g}}$ in $\left[\mathrm{N} / \mathrm{m}^{3}\right]$ the specific gravity force is $\mathbf{b}_{\mathbf{g}}=g \hat{\mathbf{e}}_{\mathbf{g}}$ in $[\mathrm{N} / \mathrm{kg}]$. Similarly $\mathbf{d}_{\mathbf{i}}$ is a specific dissipative force (i.e., force per unit mass) in $[\mathrm{N} / \mathrm{kg}]$.

One may use the following identity [22]

$$
(v \cdot \nabla) v=\frac{1}{2} \nabla(v \cdot v)-v \times(\nabla \times v)
$$

into (10) and divide the whole equation by $\rho$ to yield

$$
\frac{\partial v}{\partial t}+\frac{1}{2} \nabla(v \cdot v)-v \times(\nabla \times v)=-\frac{1}{\rho} \nabla p-\frac{1}{\rho} \nabla \cdot \tau+\mathbf{b}+\mathbf{d} .
$$

In what follows we will use the following identity

$$
\nabla\left(\frac{p}{\rho}\right)=\frac{1}{\rho} \nabla p-\frac{p}{\rho^{2}} \nabla \rho
$$

and therefore

$$
\frac{1}{\rho} \nabla p=\nabla\left(\frac{p}{\rho}\right)+\frac{p}{\rho^{2}} \nabla \rho
$$

Similarly, since usually all body forces are derived from a potential, if the total body force has a potential $V_{B}$, i.e.,

$$
\mathbf{B}=-\nabla V_{B}
$$

Then

$$
\mathbf{b}=\frac{\mathbf{B}}{\rho}=-\frac{1}{\rho} \nabla V_{B}
$$

and similarly to (18)

$$
\frac{1}{\rho} \nabla V_{B}=\nabla\left(\frac{V_{B}}{\rho}\right)+\frac{V_{B}}{\rho^{2}} \nabla \rho
$$

and when substituted into (20) it produces

$$
\mathbf{b}=-\nabla\left(\frac{V_{B}}{\rho}\right)-\frac{V_{B}}{\rho^{2}} \nabla \rho
$$


which upon substitution of (18) and (22) into (16) yields

$$
\frac{\partial v}{\partial t}+\frac{1}{2} \nabla(v \cdot v)-v \times(\nabla \times v)=-\nabla\left(\frac{p}{\rho}\right)-\frac{p}{\rho^{2}} \nabla \rho-\frac{1}{\rho} \nabla \cdot \tau-\nabla\left(\frac{V_{B}}{\rho}\right)-\frac{V_{B}}{\rho^{2}} \nabla \rho+\mathbf{d} .
$$

By introducing the notation (for $m=$ const. )

$$
U_{o}=\frac{p}{\rho} ; V=m \frac{V_{B}}{\rho} ; \sigma=\frac{1}{\rho} \nabla \cdot \tau
$$

into (23) produces the equation

$$
\frac{\partial v}{\partial t}+\underbrace{\frac{1}{2} \nabla(v \cdot v)}_{\begin{array}{c}
\text { irrotational } \\
\text { inertial part }
\end{array}}-\underbrace{v \times(\nabla \times v)}_{\begin{array}{c}
\text { rotational } \\
\text { inertial part }
\end{array}}=-\nabla U_{o}-\frac{1}{m} \nabla V-\frac{1}{\rho}\left(U_{o}+\frac{V}{m}\right) \nabla \rho-\sigma+\mathbf{d}
$$

where the rotational and irrotational components of the inertial terms are identified.

\subsection{Helmholtz Decomposition}

Introducing the Helmholtz decomposition for $v$ in the form

$$
v=-\nabla \varphi+\nabla \times x
$$

where $\varphi$ is a scalar potential defined up to an arbitrary constant (i.e., one may add any arbitrary constant $C_{0}$ to $\varphi$ without altering the value of $v$ ), and $\chi$ is a vector potential defined up to a gradient of an arbitrary function (i.e., one may add a gradient of any arbitrary function $f(x, y, z)$ to $x$ without altering the value of $v$ because $\nabla \times \nabla f=0$ identically, [22]). The special case when $\chi=0$ was used by Madelung $[14,15]$, which led to rendering the Schrödinger equation into the Euler equations for potential irrotational flow, or back. In this paper an attempt is made to generalize the latter and remove the potential flow restriction, start with the general Navier-Stokes equations and obtain a modified version of the Schrödinger equation.

Then, applying the Helmholtz decomposition to the continuity Equation (11) by replacing $v$ with the right hand side of Equation (26) leads to

$$
\frac{\partial \rho}{\partial t}-\nabla \cdot(\rho \nabla \varphi)+(\nabla \rho) \cdot(\nabla \times \chi)=0
$$

where use has been made of the fact that $\nabla \cdot(\nabla \times \chi)=0$, due to the identity that the divergence of any curl of a vector function vanishes [22]. Applying the Helmholtz decomposition (26) to the momentum Equation (25) by replacing $v$ with the right hand side of Equation (26) yields

$$
\begin{gathered}
\nabla \quad[-\frac{\partial \varphi}{\partial t}+\underbrace{\frac{1}{2}(\nabla \varphi) \cdot(\nabla \varphi)+\frac{1}{2}(\nabla \times \chi) \cdot(\nabla \times \chi)-(\nabla \varphi) \cdot(\nabla \times \chi)}_{\begin{array}{c}
\text { irrotational } \\
\text { inertial part }
\end{array}}+U_{o}+\frac{1}{m} V]= \\
-\frac{\partial}{\partial t}(\nabla \times \chi)-\underbrace{(\nabla \varphi) \times(\nabla \times \nabla \times \chi)+(\nabla \times \chi) \times(\nabla \times \nabla \times \chi)}_{\begin{array}{c}
\text { rotational } \\
\text { inertial part }
\end{array}}-\frac{1}{\rho}\left(U_{o}+\frac{V}{m}\right) \nabla \rho-\sigma+\mathbf{d}
\end{gathered}
$$


where use has been made of the identity $\nabla \times(\nabla \varphi)=0$ from [22], and we moved all the terms that can be placed under a common $\nabla$ operator to the left hand side, and the rest to the right hand side. The terms under the gradient operator can be regarded as conservative terms, while the terms on the right hand side can be regarded as "apparently non-conservative terms" (a.n.c.t.), although we do not exclude the possibility that upon a further more detailed analysis it may turn out that these terms may have conservative components. Let us use for simplicity the notation for the right hand side of (28)

$$
\begin{array}{rc}
\text { a.n.c.t. }=-\frac{\partial}{\partial t}(\nabla \times \chi)-(\nabla \varphi) \times(\nabla \times \nabla \times \chi)+(\nabla \times & \chi) \times(\nabla \times \nabla \times \chi)- \\
\frac{1}{\rho}\left(U_{o}+\frac{V}{m}\right) \nabla \rho-\sigma+\mathbf{d}
\end{array}
$$

simplifying the presentation of (28) in the form

$$
\nabla[-\frac{\partial \varphi}{\partial t}+\underbrace{\frac{1}{2}(\nabla \varphi) \cdot(\nabla \varphi)+\frac{1}{2}(\nabla \times \chi) \cdot(\nabla \times \chi)-(\nabla \varphi) \cdot(\nabla \times \chi)}_{\begin{array}{c}
\text { irrotational } \\
\text { inertial part }
\end{array}}+U_{o}+\frac{1}{m} V]=\text { a.n.c.t. }
$$

Applying now on Equation (30) the inverse gradient operator $\nabla^{-1}$ defined by Equation (53) in Appendix A produces the following equation

$$
-\frac{\partial \varphi}{\partial t}+\frac{1}{2}(\nabla \varphi) \cdot(\nabla \varphi)+\frac{1}{2}(\nabla \times \chi) \cdot(\nabla \times \chi)-(\nabla \varphi) \cdot(\nabla \times \chi)+U_{o}+\frac{1}{m} V=\nabla^{-1} \text { (a.n.c.t.) }
$$

\section{Inverse Madelung Transformation and the Extended Schrödinger Equation}

Introducing the following notation that represents the inverse Madelung transformation

$$
\begin{gathered}
\varphi=-\frac{S}{m}=i \frac{\hbar}{2 m} \ln \left(\frac{\psi}{\psi^{*}}\right) \\
\rho=R^{2}=\psi \psi^{*}
\end{gathered}
$$

and

$$
U_{1}=-\frac{\hbar^{2}}{2 m^{2}} \frac{\nabla^{2} R}{R}
$$

where $R$ and $S$ are the radius and phase-related variables in the polar representation of the complex wave function $\psi$ in Equation (5), and $m=$ const. This inverse transformation followed by the adopted procedure will render the scalar and vector variables of density $\rho$ and velocity $v$, respectively, from the continuity and momentum equations into the complex scalar wave function $\psi$ of the Schrödinger equation. Substituting this inverse transformation (32), (33) and (34) into the continuity Equation (27) and the momentum Equation (31) yields for the continuity equation

$$
2 R \frac{\partial R}{\partial t}+\frac{1}{m} \nabla \cdot\left(R^{2} \nabla S\right)+2 R \nabla R \cdot(\nabla \times x)=0 .
$$

By using the first identity proven in Appendix A, Equation (56) into Equation (35) and dividing the whole equation by $2 R$ produces the result

$$
\frac{\partial R}{\partial t}=-\frac{1}{2 m}\left[R \nabla^{2} S+2(\nabla R) \cdot(\nabla S)\right]-\nabla R \cdot(\nabla \times \chi) .
$$


For the momentum equation after applying the transformation (32) and (33), assuming that $U_{o}=U_{1}$, and multiplying the whole equation by $m$ one obtains

$$
\frac{\partial S}{\partial t}=-\frac{1}{2 m}\left[(\nabla S) \cdot(\nabla S)-\hbar^{2} \frac{\nabla^{2} R}{R}\right]-(\nabla S) \cdot(\nabla \times \chi)-V-\frac{m}{2}(\nabla \times \chi) \cdot(\nabla \times \chi)+m \nabla^{-1} \text { (a.n.c.t.) }
$$

The next step is combining the continuity (36) and momentum (37) equations, by multiplying Equation (36) by $(i \hbar \exp [i S / \hbar])$ and (37) by $(-R \exp [i S / \hbar])$, where $i=\sqrt{-1}$, which leads to the following two equations

$$
\begin{gathered}
i \hbar e^{\frac{i}{\hbar} S} \frac{\partial R}{\partial t}=-\frac{i \hbar}{2 m} e^{\frac{i}{\hbar} S}\left[R \nabla^{2} S+2(\nabla R) \cdot(\nabla S)+2 m \nabla R \cdot(\nabla \times x)\right] \\
-R e^{\frac{i}{\hbar} S} \frac{\partial S}{\partial t}=-\frac{\hbar^{2}}{2 m} R e^{\frac{i}{\hbar} S}\left[\frac{\nabla^{2} R}{R}-\frac{1}{\hbar^{2}}(\nabla S) \cdot(\nabla S)\right]+ \\
R e^{\frac{i}{\hbar} S}(\nabla S) \cdot(\nabla \times \chi)+R e^{\frac{i}{\hbar} S} V+\frac{m}{2} R e^{\frac{i}{\hbar} S}(\nabla \times \chi) \cdot(\nabla \times \chi)+m R e^{\frac{i}{\hbar} S} \nabla^{-1} \text { (a.n.c.t.) }
\end{gathered}
$$

Introducing now the complex notation definition that combines the two equations

$$
\psi=R \exp \left(\frac{i}{\hbar} S\right) \Rightarrow \ln \psi=\ln R+\frac{i}{\hbar} S
$$

produces the identity

$$
\frac{1}{\psi} \frac{\partial \psi}{\partial t}=\frac{\partial \ln \psi}{\partial t}=\frac{\partial \ln R}{\partial t}+\frac{i}{\hbar} \frac{\partial S}{\partial t}=\frac{1}{R} \frac{\partial R}{\partial t}+\frac{i}{\hbar} \frac{\partial S}{\partial t} .
$$

Substituting (40) into (38) and (39) yields

$$
\begin{gathered}
i \hbar \frac{\psi}{R} \frac{\partial R}{\partial t}=-\frac{i \hbar}{2 m} \frac{\psi}{R}\left[R \nabla^{2} S+2(\nabla R) \cdot(\nabla S)+2 m \nabla R \cdot(\nabla \times \chi)\right] \\
-\psi \frac{\partial S}{\partial t}=-\frac{\hbar^{2}}{2 m} \frac{\psi}{R}\left[\nabla^{2} R-\frac{R}{\hbar^{2}}(\nabla S) \cdot(\nabla S)\right]+\psi(\nabla S) \cdot(\nabla \times \chi)+\psi V+\psi \frac{m}{2}(\nabla \times \chi) \cdot(\nabla \times \chi)+ \\
\psi m \nabla^{-1} \text { (a.n.c.t.) }
\end{gathered}
$$

Adding (42) to (43)

$$
\begin{aligned}
& \psi\left[\frac{i \hbar}{R} \frac{\partial R}{\partial t}-\frac{\partial S}{\partial t}\right]= \\
& -\frac{\hbar^{2}}{2 m} \frac{\psi}{R}\left[\nabla^{2} R-\frac{R}{\hbar^{2}}(\nabla S) \cdot(\nabla S)+\frac{i}{\hbar} R \nabla^{2} S+i \frac{2}{\hbar}(\nabla R) \cdot(\nabla S)+i \frac{2 m}{\hbar} \nabla R \cdot(\nabla \times x)\right]+ \\
& \psi(\nabla S) \cdot(\nabla \times \chi)+\psi V+\psi \frac{m}{2}(\nabla \times \chi) \cdot(\nabla \times x)+\psi m \nabla^{-1} \text { (a.n.c.t.) }
\end{aligned}
$$

and using the second identity Equation (61) proven in Appendix A, and Equation (41), while using $1 / i \hbar=-i / \hbar$, transforms Equation (44) into

$$
\begin{aligned}
& \underbrace{i \hbar \frac{\partial \psi}{\partial t}=-\frac{\hbar^{2}}{2 m} \nabla^{2} \psi+\psi V}_{\text {Schrödinger Equation Terms }}- \\
& \underbrace{i \hbar \frac{\psi}{R} \nabla R \cdot(\nabla \times \chi)+\psi(\nabla S) \cdot(\nabla \times \chi)+\psi \frac{m}{2}(\nabla \times \chi) \cdot(\nabla \times \chi)+\psi m \nabla^{-1} \text { (a.n.c.t.) }}_{\text {Additional Terms }}
\end{aligned}
$$


To remove the explicit appearance of $R$ and $S$ in the additional terms of Equation (45) we can represent the first two additional terms as follows

$$
\begin{aligned}
& -i \hbar \psi \frac{\nabla R}{R} \cdot(\nabla \times x)+\psi(\nabla S) \cdot(\nabla \times x)=-i \hbar \psi\left[\nabla(\ln R)-\frac{1}{i \hbar} \nabla S\right] \cdot(\nabla \times \chi) \\
& =-i \hbar \psi\left[\nabla(\ln R)+\frac{i}{\hbar} \nabla S\right] \cdot(\nabla \times \chi)= \\
& -i \hbar \psi \nabla\left[\ln R+\frac{i}{\hbar} S\right] \cdot(\nabla \times \chi) \underbrace{=}_{(40)}-i \hbar \psi(\nabla \ln \psi) \cdot(\nabla \times \chi)=-i \hbar(\nabla \psi) \cdot(\nabla \times \chi)
\end{aligned}
$$

producing the final form of the extended Schrödinger equation

$$
\underbrace{i \hbar \frac{\partial \psi}{\partial t}=-\frac{\hbar^{2}}{2 m} \nabla^{2} \psi+V \psi}_{\text {Schrödinger Equation Terms }}-\underbrace{\underbrace{(\text { a) }}_{i \hbar(\nabla \psi) \cdot(\nabla \times \chi)}+\overbrace{\frac{m}{2}(\nabla \times \chi) \cdot(\nabla \times \chi)}+\overbrace{\psi m \nabla^{-1} \text { (a.n.c.t.) }}^{(b)}}_{\text {Additional Terms }}
$$

The first three terms in the equation represent the original Schrödinger equation, while the additional terms labeled (a), (b) and (c), are the result of converting the complete Navier-Stokes equations leading to an extended form of Schrödinger equation.

\section{Results and Discussion}

We shall be attempting now to identify these additional terms by seeking their origin.

Term (a) can be tracked down to the fluid "compressibility" in the continuity equation, i.e., from the term (see Equation (27))

$$
\nabla \cdot(\rho \nabla \times \chi)=\rho \underbrace{\nabla \cdot(\nabla \times \chi)}_{=0}+(\nabla \rho) \cdot(\nabla \times \chi)=(\nabla \rho) \cdot(\nabla \times \chi)
$$

and more accurately it results from the combination of fluid's compressibility and rotationality, i.e., the fact that $\nabla \rho \neq 0$ and $(\nabla \times \chi) \neq 0$, combined with a rotational term from the momentum equation, part of the contribution of the vector potential to the kinetic energy. It is a nonlinear term in $\psi$, transforming the extended Schrödinger equation into a nonlinear equation even before considering contributions from the momentum equation via the other additional terms.

Term (b) is linked to the contribution of the vector potential of $v$ from Equation (26), i.e., $(\nabla \times x)$ to the kinetic energy, which can be observed when presenting the latter in the form

$$
\begin{aligned}
& \frac{1}{2} m v \cdot v=\frac{1}{2} m(-\nabla \varphi+\nabla \times \chi) \cdot(-\nabla \varphi+\nabla \times \chi)= \\
& \frac{1}{2} m(\nabla \varphi) \cdot(\nabla \varphi)+\underbrace{\frac{1}{2} m(\nabla \times \chi) \cdot(\nabla \times \chi)}_{\text {term (b) contribution }}-\underbrace{m(\nabla \varphi) \cdot(\nabla \times \chi)}_{\text {part of term (a) contribution }}
\end{aligned}
$$

Term (c) is a combination of terms that were apparently non-conservative. Its specific term-by-term representation by using its definition from (29) takes the following form

$$
\begin{aligned}
\nabla^{-1}(\text { a.n.c.t. })= & -\frac{\partial}{\partial t} \nabla^{-1}(\nabla \times \chi)-\nabla^{-1}[(\nabla \varphi) \times(\nabla \times \nabla \times \chi)]+\nabla^{-1}[(\nabla \times \chi) \times(\nabla \times \nabla \times \chi)]- \\
& \nabla^{-1}\left[\frac{1}{\rho}\left(U_{o}+\frac{V}{m}\right) \nabla \rho\right]-\nabla^{-1} \sigma+\nabla^{-1} \mathbf{d}
\end{aligned}
$$

Quantum theory does not usually include dissipating effects in its treatment of sub-atomic phenomena. Therefore, these apparently non-conservative terms which by their nature lead to dissipative effects are obviously excluded from the quantum mechanics description and consequently are absent from the original Schrödinger equation. Further analysis may lead to additional simplifications in the presentation of these apparently non-conservative terms lumped together in term (c). 
The main result derived in this paper is the fact that the Navier-Stokes equations for a compressible fluid were shown to render into an extended generalized version of the Schrödinger equation, where the first three terms are identical to the original Schrödinger equation from quantum mechanics. No assumptions of potential flow were necessary. The latter are required when converting the Schrödinger equation into the Euler equations for a compressible fluid.

\section{Conclusions}

The fact that Navier-Stokes equations for compressible flow can be rendered into an extended generalized version of Schrödinger equation applicable to sub-atomic phenomena in quantum mechanics suggests the possibility that quantum effects at sub-atomic levels deal with a compressible fluid susceptible to wave propagation, rather than a particle. The link between such a fluid and the quantum "particle" is under current investigation. It therefore indicates that the EPR [10] conclusion that a "theory providing a complete description of the physical reality might be possible" cannot be excluded. However, such a theory has not yet been developed.

Conflicts of Interest: The author declares no conflict of interest.

\section{Abbreviations}

The following abbreviations are used in this manuscript:

EPR Einstein, Podolsky, and Rosen paper [10]

\section{Appendix A Appendix A: Definition of the Inverse Gradient Operator}

The gradient operator is defined in Cartesian coordinates in the form

$$
\nabla \equiv \frac{\partial}{\partial x} \hat{\mathbf{e}}_{\mathbf{x}}+\frac{\partial}{\partial y} \hat{\mathbf{e}}_{\mathbf{y}}+\frac{\partial}{\partial z} \hat{\mathbf{e}}_{\mathbf{z}}
$$

When operating on a scalar function $f(x, y, z)$ it produces the vector result

$$
\nabla f \equiv \frac{\partial f}{\partial x} \hat{\mathbf{e}}_{\mathbf{x}}+\frac{\partial f}{\partial y} \hat{\mathbf{e}}_{\mathbf{y}}+\frac{\partial f}{\partial z} \hat{\mathbf{e}}_{\mathbf{z}}
$$

Then, one may define the inverse gradient operator for Cartesian coordinates in the form

$$
\nabla^{-1} \equiv \int \mathrm{d} x \hat{\mathbf{e}}_{\mathbf{x}} \cdot+\int \mathrm{d} y \hat{\mathbf{e}}_{\mathbf{y}} \cdot+\int \mathrm{d} z \hat{\mathbf{e}}_{\mathbf{z}}
$$

where the dots $(\cdot)$ represent the scalar product, and operating (A3) on (A2) yields

$$
\begin{aligned}
\nabla^{-1} \nabla f \equiv & \int \mathrm{d} x\left(\frac{\partial f}{\partial x}\right) \hat{\mathbf{e}}_{\mathbf{x}} \cdot \hat{\mathbf{e}}_{\mathbf{x}}+\int \mathrm{d} y\left(\frac{\partial f}{\partial y}\right) \hat{\mathbf{e}}_{\mathbf{y}} \cdot \hat{\mathbf{e}}_{\mathbf{y}}+\int \mathrm{d} z\left(\frac{\partial f}{\partial z}\right) \hat{\mathbf{e}}_{\mathbf{z}} \cdot \hat{\mathbf{e}}_{\mathbf{z}}= \\
& \int\left[\left(\frac{\partial f}{\partial x}\right) \mathrm{d} x+\left(\frac{\partial f}{\partial y}\right) \mathrm{d} y+\left(\frac{\partial f}{\partial z}\right) \mathrm{d} z\right]=\int \mathrm{d} f=f
\end{aligned}
$$

producing the result

$$
\nabla^{-1} \nabla f=f
$$

and proving that indeed the $\nabla^{-1}$ defined by Equation (A3) is the inverse of a gradient operator. The operator $\nabla^{-1}$ when operating on a vector yields a scalar result. 


\section{Appendix A Appendix B: The First Identity}

In this appendix the proof of the following identity is provided

$$
\nabla \cdot\left(R^{2} \nabla S\right)=R\left[R \nabla^{2} S+2(\nabla R) \cdot(\nabla S)\right]
$$

Proof: Using the following vector operators identity [22] applicable to any scalar $U$ and vector $\mathbf{A}$

$$
\nabla \cdot(U \mathbf{A})=\nabla U \cdot \mathbf{A}+U \nabla \cdot \mathbf{A}
$$

and setting

$$
U \equiv R^{2} \text { and } \mathbf{A}=\nabla S
$$

one obtains after substituting (B3) into (B2) the following result

$$
\begin{aligned}
\nabla \cdot\left(R^{2} \nabla S\right)= & \nabla R^{2} \cdot \nabla S+R^{2} \nabla \cdot \nabla S= \\
& 2 R(\nabla R) \cdot(\nabla S)+R^{2} \nabla^{2} S=R\left[R \nabla^{2} S+2(\nabla R) \cdot(\nabla S)\right]
\end{aligned}
$$

\section{Appendix A Appendix C: The Second Identity}

In this appendix the proof of the following identity is provided

$$
\nabla^{2} \psi=e^{\frac{i}{\hbar} S}\left[\nabla^{2} R-\frac{R}{\hbar^{2}}(\nabla S) \cdot(\nabla S)+\frac{i}{\hbar} R \nabla^{2} S+i \frac{2}{\hbar}(\nabla R) \cdot(\nabla S)\right]
$$

Where

$$
\psi=R \exp \left(\frac{i}{\hbar} S\right)
$$

Proof:

$$
\begin{aligned}
& \nabla^{2} \psi=\nabla^{2}\left[R e^{\frac{i}{\hbar} S}\right]=\nabla \cdot \nabla\left[R e^{\frac{i}{\hbar} S}\right]=\nabla \cdot\left[R \nabla e^{\frac{i}{\hbar} S}+e^{\frac{i}{\hbar} S} \nabla R\right]= \\
& \nabla \cdot\left[R \frac{i}{\hbar} e^{\frac{i}{\hbar} S} \nabla S+e^{\frac{i}{\hbar} S} \nabla R\right]=\nabla \cdot\left[e^{\frac{i}{\hbar} S}\left(\frac{i}{\hbar} R \nabla S+\nabla R\right)\right]= \\
& e^{\frac{i}{\hbar} S} \nabla \cdot\left[\frac{i}{\hbar}(R \nabla S)+\nabla R\right]+\left[\nabla e^{\frac{i}{\hbar} S}\right] \cdot\left(\frac{i}{\hbar} R \nabla S+\nabla R\right)= \\
& e^{\frac{i}{\hbar} S}\left[\frac{i}{\hbar} \nabla \cdot(R \nabla S)+\nabla^{2} R\right]+\frac{i}{\hbar} e^{\frac{i}{\hbar} S} \nabla S \cdot\left(\frac{i}{\hbar} R \nabla S+\nabla R\right)= \\
& e^{\frac{i}{\hbar} S}\left\{\frac{i}{\hbar}\left[R \nabla^{2} S+\nabla R \cdot \nabla S\right]+\nabla^{2} R+\frac{i^{2}}{\hbar^{2}} \nabla S \cdot(R \nabla S)+\frac{i}{\hbar} \nabla S \cdot \nabla R\right\}= \\
& e^{\frac{i}{\hbar} S}\left[\nabla^{2} R-\frac{R}{\hbar^{2}}(\nabla S) \cdot(\nabla S)+\frac{i}{\hbar} R \nabla^{2} S+i^{2}(\nabla R) \cdot(\nabla S)\right]
\end{aligned}
$$

\section{References}

1. Bohm, D. Quantum Theory; Dover Publications: Mineola, NY, USA, 1989.

2. Griffiths, D.J. Introduction to Quantum Mechanics, 2nd ed.; Pearson Prentice Hall: Upper Saddle River, NJ, USA, 2005.

3. Bowman, G.E. Essential Quantum Mechanics; Oxford University Press: Oxford, UK, 2008.

4. Schrödinger, E. Quantisierung als Eigenwertproblem (Erste Mitteilung). Ann. Phys. 1926, 79, 361-376. [CrossRef]

5. Schrödinger, E. Quantisierung als Eigenwertproblem (Zweite Mitteilung). Ann. Phys. 1926, 79, 489-527. [CrossRef]

6. Schrödinger, E. Quantisierung als Eigenwertproblem (Dritte Mitteilung: Störungstheorie, mit Anwendung auf den Starkeffekt der Balmerlinien). Ann. Phys. 1926, 80, 437-490. 
7. Schrödinger, E. Quantisierung als Eigenwertproblem (Vierte Mitteilung). Ann. Phys. 1926, 81, $109-139$. [CrossRef]

8. Schrödinger, E. Collected Papers on Wave Mechanics, 3rd (augmented) English Edition; AMS Chelsea Publishing Company: New York, NY, USA, 1982.

9. Born, M. Zur Quantenmechanik der Stoßvorgänge. Z. Phys. 1926, 37, 863-867. [CrossRef]

10. Einstein, A.; Podolsky, B.; Rosen, N. Can Quantum-Mechanical Description of Physical Reality be Considered Complete? Phys. Rev. 1935, 47, 777-780. [CrossRef]

11. Einstein, A. Zur Theorie der Brownschen Bewegung. Ann. Phys. 1906, 19, 371-381. [CrossRef]

12. Einstein, A. On the Theory of the Brownian Movement. In Albert Einstein: Investigations on the Theory of Brownian Movement; Dover Publications: Mineola, NY, USA, 1956; pp. 19-35.

13. Bohr, N. Can Quantum-Mechanical Description of Physical Reality be Considered Complete? Phys. Rev. 1935, 48, 696-702. [CrossRef]

14. Madelung, E. Quantentheorie in hydrodynamischer Form. Z. Phys. 1926-1927, 40, 322-326. [CrossRef]

15. Madelung, E. Quantum Theory in Hydrodynamical Form. Available online: http://www.neoclassical-physics.info/uploads/3/0/6/5/3065888/madelung_-_hydrodynamical_interp..pdf (accessed on 12 June 2016).

16. Sonego, D. Interpretation of the Hydrodynamical Formalism of Quantum Mechanics. Found. Phys. 1991, 21, 1135-1181. [CrossRef]

17. Takabayasi, T. On the Formulation of Quantum Mechanics associated with Classical Pictures. Prog. Theor. Phys. 1952, 8, 143-182. [CrossRef]

18. Takabayasi, T. Remarks on the Formulation of Quantum Mechanics with Classical Pictures and on Relations between Linear Scalar Fields and Hydrodynamical Fields. Prog. Theor. Phys. 1952, 9, 187-222. [CrossRef]

19. Wilhelm, H.E. Hydrodynamic Model of Quantum Mechanics. Phys. Rev. D 1970, 1, 2278-2285. [CrossRef]

20. Sorokin, A.L. Madelung Transformations for Vortex Flows of a Perfect Fluid. Dokl. Phys. 2001, 46, 576-578. [CrossRef]

21. Broadbridge, P. Classical and Quantum Burgers Fluids: A Challenge for Group Analysis. Symmetry 2015, 7, 1803-1815. [CrossRef]

22. Spiegel, M.R.; Lipschutz, S.; Liu, J. Mathematical Handbook of Formulas and Tables; Schaum's Outline Series; McGraw Hill: New York, NY, USA, 2009. 